\title{
Based on GSM Automated and Smart Irrigation System Using Android
}

\author{
J. Ravichandran \\ Lecturer Senior Grade, Department of Instrumentation and Control Engineering, \\ Sri Krishna Polytechnic College, Coimbatore, Tamil Nadu, India
}

\begin{abstract}
The greenhouse based modern agriculture industries are the recent requirement in every part of agriculture in India. In this technology, the humidity and temperature of plants are precisely controlled. Due tothe variable atmospheric circumstances these conditions sometimes may vary from place to place in large farmhouse, which makes very difficult to maintain the uniformity at all the places in the farmhouse manually. It is observed that for the first time an android phone-control the Irrigation system, which could give the facilities of maintaining uniform environmental conditions are proposed. The Android Software Development Kit provides the tools and Application Programmable Interface necessary to begin developing applications on the Android platform using the Java programming language. Mobile phones have almost become an integral part of human life serving multiple needs of humans. This application makes use of the GPRS [General Packet Radio Service] feature of mobile phone as a solution for irrigation control system.GSM (Global System for Mobile Communication) is used to inform the user about the exact field condition. The information is passed onto the user request in the form of SMS.
\end{abstract}

Keywords: GSM module (mobile), Android, SMS, automatic irrigation, microcontroller, Temperature sensor, soil moisture sensor, regulated power supply.

\section{INTRODUCTION:}

Irrigation is a scientific process of artificially supplying water to the land or soil that is being cultivated. Traditionally in dry regions having no or little rainfall water had to be supplied to the fields either through canals or hand pumps, tube wells. Conventional irrigation methods had severe problems such as increase in workload off arm labor and often it lead to problem such as over irrigation or under-irrigation, and leaching of soil. Develop android based automatic Farming system capable of controlling many electrical appliances in an irrigation or field using android platform with a mobile handset, where data transmission is carried wirelessly. That's why design Wireless transmission media using Wi-Fi transceivers and its interfacing peripherals for wireless data communication between Mobile Handset and appliances is our need. Hence to create a database of user interface in order to characterize the electric signals to atomize farming system. And to develop the GUI interface to monitor and change the current status of field on any android smart phones. Another important point is not only monitor the temperature and maintain moisture level in the field for proper growth of plants but also save water, Energy and man power in the agriculture Sector. So we design such a system that will be efficient and effort reducing of the farmer. Hence we design the System which is operated manually as well as automatically from remote locations by using Android.

The GSM based irrigation system [Fig.1] may offer users the flexibility to regulate and control the operations of their irrigation systems with little intervention to reduce runoff from over watering for improvement in crop yield. This enables users to take advantage of the globally deployed GSM networks with its low SMS service cost to use mobile phones and simple SMS commands to manage their irrigation system. It will be possible for users to use SMS to monitor directly the conditions of their farmland, schedule the water needs of crops, automatically control watering, and set control operational conditions in accordance with the water needs of 
crops. This will help minimize overwatering and crop production cost. Drip irrigation is artificial method of supplying water to the roots of the plant. It is also called micro irrigation. In past few years there is a rapid growth in this system.

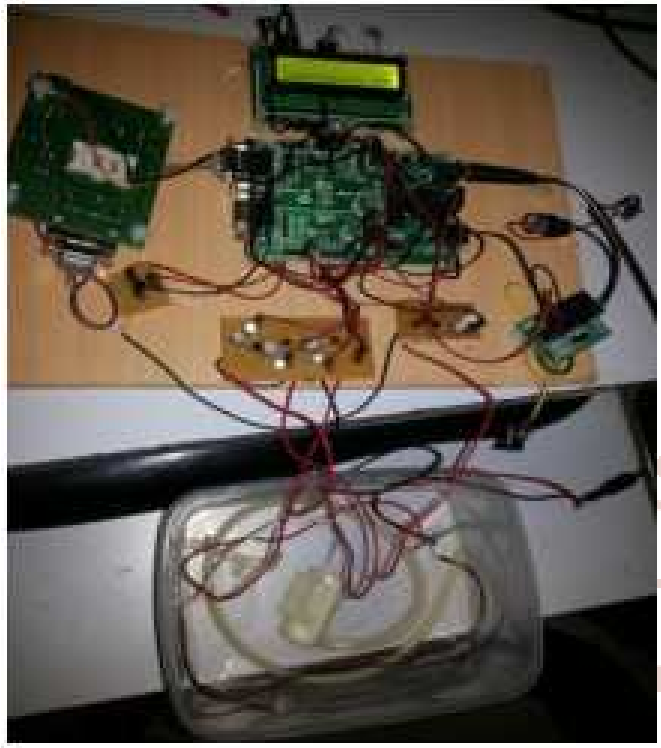

Fig.1: GSM based automatic irrigation control system

\section{Android Architecture}

Android is a mobile operating system that is based on a modified version of Linux. It was originally developed by a start up of the same name, Android, Inc. In 2005, as part of its strategy to enter the mobile space, Google purchased Android and took over its development work (as well as its development team).The followingFig.2 shows the major components of the Android operating system. The main advantage of adopting Android is that it offers a unified approach to application development and their applications should be able to run on numerous different devices, as long as the devices are powered using Android Applications: These are applications written in Java. Some of basic applications include calendar, email client, SMS program, maps, making phone calls, accessing the Web browser, accessing your contacts list and others.

\section{Application Framework:}

This is the skeleton or framework which all android developers have to follow. The developers can access all framework APIs an manage phone's basic functions like resource allocation, switching between processes or programs, telephone applications, and keeping track of the phone's physical location.
GSM:

The Global System for Mobile Communication is a standard set developed by the European Telecommunications Standards Institute (ETSI) to describe technologies ( $2 \mathrm{G}$ and $3 \mathrm{G}$ ).

\section{Libraries:}

This layer consists of Android libraries written in C, $\mathrm{C}++$, and used by various systems. These libraries tell the device how to handle different kinds of data and are exposed to Android developers via Android Application framework. Some of these libraries include media, graphics, 3D, SQL, web browser library etc. The Android runtime layer which includes set of core java libraries and DVM (Dalvik Virtual Machine) is also located in same layer.

\section{Runtime Android:}

This layer includes set of base libraries that are required for java libraries. Every Android application gets its own instance of DVM. Dalvik has been written so that a device can run multiple VMs efficiently and it executes files in executable (.Dex) optimized for minimum memory.

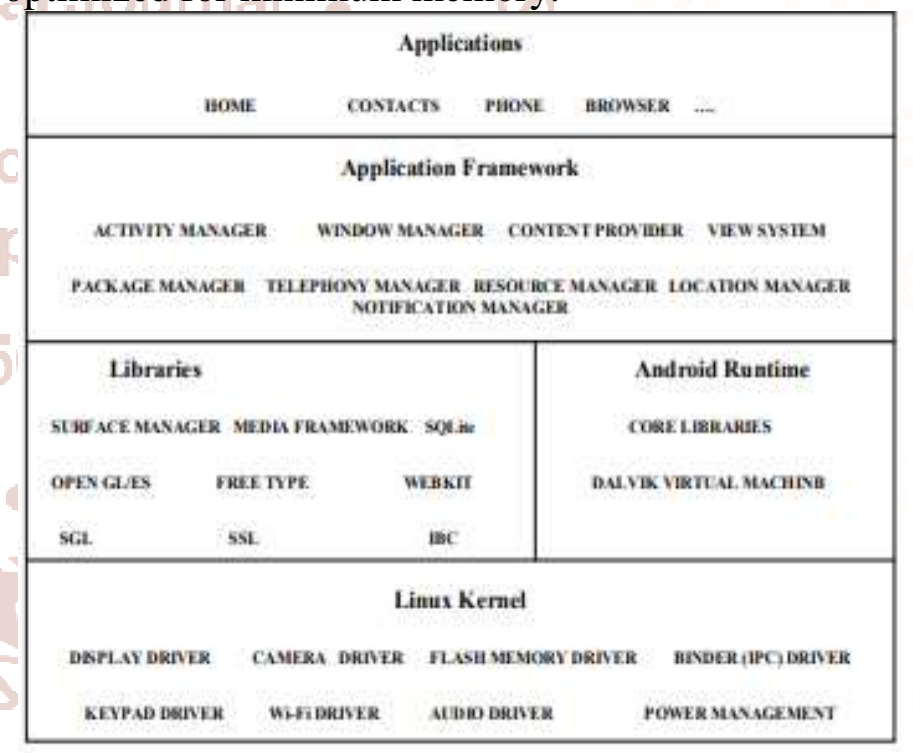

Fig.2: Android Architecture

\section{Kernel Linux:}

This layer includes Android's memory management programs, security settings, power management software and several drivers for hardware, file system access, networking and inter-process communication. The kernel also acts as an abstraction layer between hardware and the rest of the software stack. 
III. GSM Based Irrigation Control System

The connections between the two mobiles are done using GSM. The GSM module and microcontroller are connected using UART (universal asynchronous receiver / transmitter). When the moisture sensor senses the low moisture content of the soil, it gives a signal to the microcontroller. The microcontroller then gives a signal to the called mobile (which is kept in the auto answering mode). The called mobile activates the buzzer.

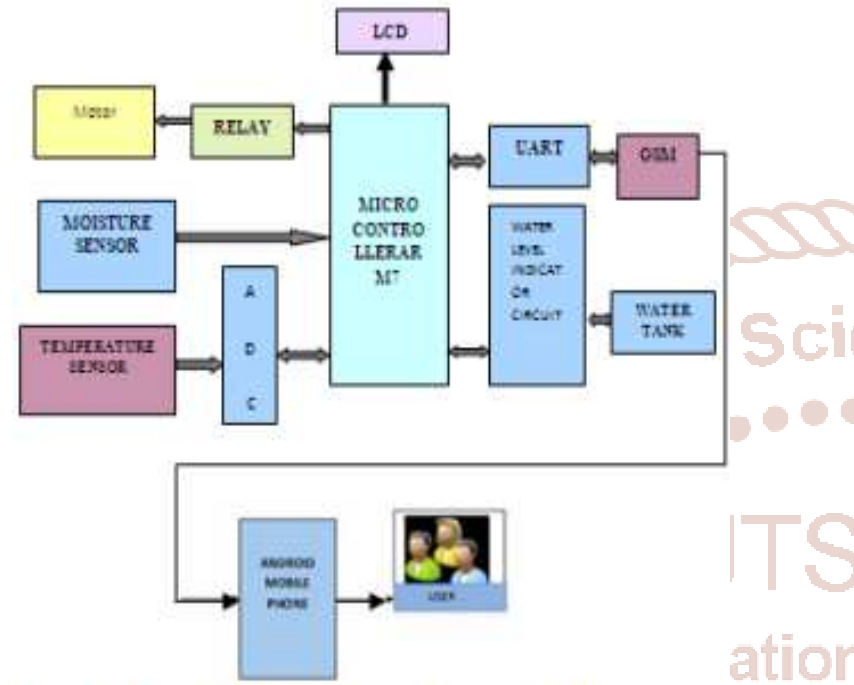

Fig.3: Block Diagram of the Irrigation Control System consumption. The ARM architecture is based on Reduced Instruction Set Computer (RISC) principles, and the instruction set and related decode mechanism are much simpler than those of micro programmed Complex Instruction Set Computers Can be used to control water flow.

\section{Structure of GSM Module}

At present the GSM module is used for Remote Control activities such as Gate Control, Temperature Control etc. GSM/GPRS module consists of a GSM/GPRS modem assembled together with power supply circuit and communication interfaces (like RS232, USB) for computer. The MODEM is the soul of such modules. They generate, transmit or decode data from a cellular network, for establishing communication between the cellular network and the computer. These are manufactured for specific cellular network (GSM/UMTS/CDMA) or specific cellular data standard (GSM/UMTS/GPRS/EDGE/HSDPA) or technology (GPS/SIM). They use serial communication to interface with the user and need Hayes compatible AT (Attention) commands for communication with the computer (any microprocessor or microcontroller system).

Therefore when calling mobile calls, that buzzer is heard indicating the valve needs to be open. By pressing the button in the called function, the signal is given back to the microcontroller. The microcontroller gives signal to the valves which causes it to get open. The water is given to the root of the plant drop by drop, and when the moisture content becomes sufficient, the sensor senses this and gives back the signal to the Applications microcontroller and the buzzer becomes off. Then by pressing the button in the calling function again, the valve is made off. The power supply needed by the controlling system is $+5 \mathrm{~V}$. The entire unit is as shown in Fig.3.

An UART is responsible for performing the main task in serial communications with computers. The device changes incoming parallel information to serial data which can be sent on a communication line. A second UART can be used to receive the information. The UART performs all the tasks, timing, parity checking, etc. needed for the communication. The only extra devices attached are line driver chips capable of transforming the TTL level signals to line voltages and vice versa. The Microcontroller ARM7 structure is a general purpose 32-bit microprocessor, which offers high performance and very low power
An RS-232 port was once a standard feature of a personal computer for connections to modems, printers, mice, data storage, un-interruptible power supplies, and other peripheral devices. However, the limited transmission speed, relatively large voltage swing, and large standard connectors motivated development of the universal serial bus which has displaced RS-232 from most of its peripheral interface roles. Many modern personal computers have no RS232 ports and must use an external converter to connect to older peripherals. Some RS-232 devices are still found especially in industrial machines or scientific instruments.

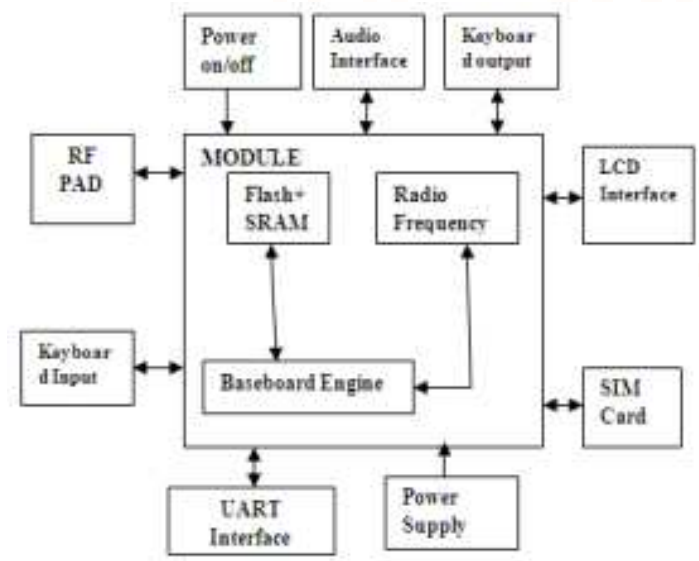

Fig.4: GSM module structure 


\section{Liquid/moisture sensor and precision centigrade temperature sensors}

Detects presence of liquid or moisture between two wire leads and gives active High output. The exposed wire is porous; therefore it allows transmission of water vapors into the sensor. These exposed areas are engineered very thinly. Therefore the sensor responds very rapidly to changes in applied moisture, both when being dried (on process start-up) and when called into action if there is moisture ingress into a process.

These are the types of sensors mainly used for,

$>$ Interfacing with Microcontroller to detect liquid levels.

$>$ Moisture detection for automatic watering of plants.

$>$ Liquid level detection by putting multiple probes at each liquid level.

Another important feature of this circuit is induction of Precision Centigrade Temperature Sensors. TheLM35 is an integrated circuit sensor that can be used to measure temperature with an electrical output proportional to the temperature (in ${ }^{\circ} \mathrm{C}$ ). With an LM35, temperature can be measured more accurately than using a thermostat. The sensor circuitry is sealed and not subject to oxidation.

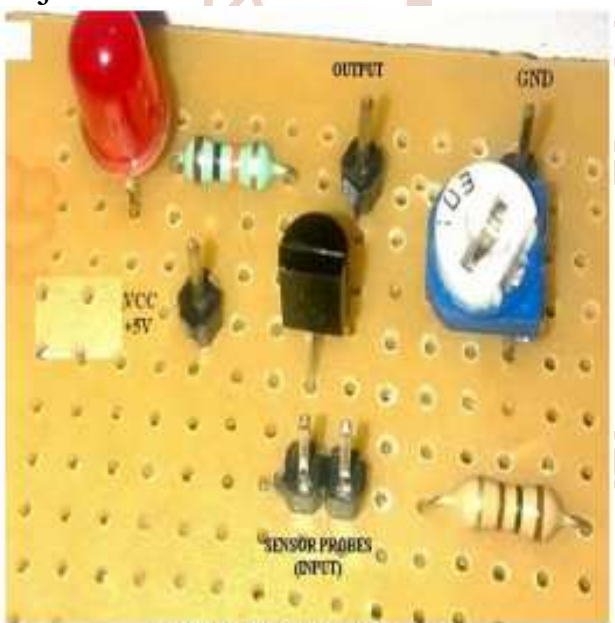

Fig.5: Moisture Sensor

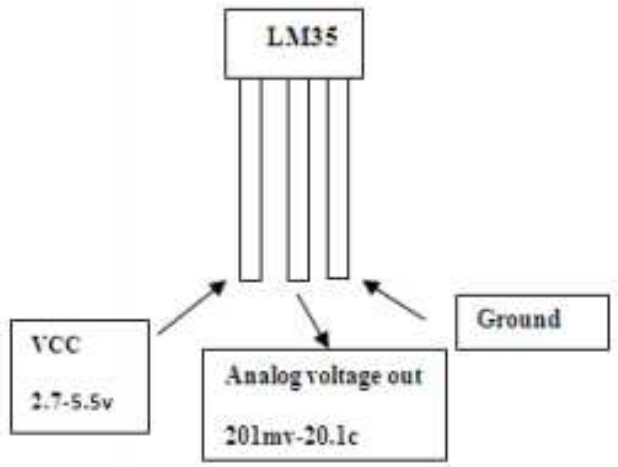

Fig.6: Precision Centigrade Temperature Sensors

\section{Water level indicator}

The model consists of a series of tanks arranged one below the other. The volume of the tanks is in descending order. Water flows from the top tank through outlets at the bottom. Three tanks or tropic levels chosen for the model is the optimum number required to analyze the effect of top down and bottom up controls.

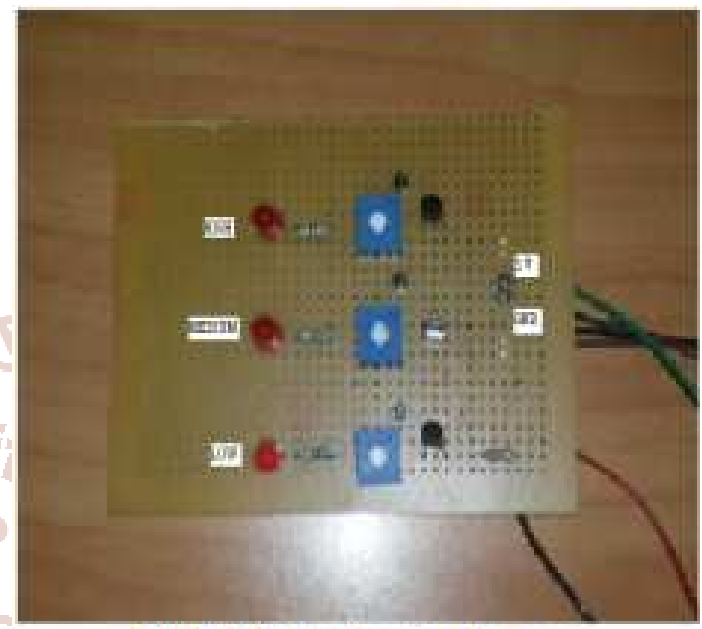

Fig7: Water level indicator

\section{Software Implementations}

Android Software development kit

Android software development is the process by which new applications are created for the Android operating system. Applications are usually developed in the Java programming language using the Android Software Development Kit. The Android software development kit (SDK) includes a comprehensive set of "development tools. These include a debugger, libraries, a handset emulator based on QEMU, documentation, sample code, and tutorials. The SDK also supports older versions of the Android platform in case developers wish to target their applications at older devices. Development tools are downloadable components, so after one has downloaded the latest version and platform, older platforms and tools can also be downloaded for compatibility testing. Android applications are packaged in.apk format and stored under /data/app folder on the Android OS (the folder is accessible only to the root user for security reasons). APK package contains.dex files (compiled byte code files called Dalvik executable), resource files, etc...

\section{Conclusions}

Irrigation has been the backbone of human civilization since man has started agriculture. As the generation evolved, man developed many methods of irrigation to supply water to the land. In the present 
scenario on conservation of water is of high importance. Present work is attempts to save the natural resources available for human kind. By continuously monitoring the status of the soil, we can control the flow of water and thereby reduce the wastage. By knowing the status of moisture and temperature through GSM with the use of moisture and temperature sensors, water flow can be controlled by just sending a message from our mobile. Conservation of water and labor: Since the systems are automatic, they do not require continuous monitoring by labor. System and operational flexibility: As desired, any valve can be controlled along with the pump and increases the efficiency of water use. If water is stored in tanks at irrigation lands, one can get the status of the status of the water level, temperature sensor and moisture content in soil through SMS generator by microcontroller present at the irrigation land. The system has an incorporated Bluetooth for remote monitoring which reduces the problem of range with GSM network and saves SMS cost for the farmer. The smoke sensors used to send emergency information to user in case of fire in field or burning of motor. The design is low power, low cost, small size, robust and highly versatile.

Thus, this system avoids over irrigation, under irrigation, top soil erosion and reduce the wastage of water. The main advantage is that the system's action can be changed according to the situation (crops, weather conditions, soil etc.). By implementing this system, agricultural, horticultural lands, parks, gardens, golf courses can be irrigated. Thus, this system is cheaper and efficient when compared to other type of automation system. In large scale applications, high sensitivity sensors can be implemented for large areas of agricultural lands. A stand by battery or solar cells can be implemented which comes into use in case of power cuts. A secondary pump can be used in case of failure of the pump.

\section{References}

1. Kay, M., "Smallholder irrigation technology: Prospects for sub-Saharan Africa" International Program for Technology and Research in Irrigation and Drainage, FAO, Rome, 2001, pp. 125.

2. N. Shah and I. Das, "Precision Irrigation Sensor Network Based Irrigation", a book on Problems,
Perspectives and Challenges of Agricultural Water Management, IIT Bombay, India, pp. 217-232, April 2008

3. Fangmeier, D. D., Garrot, D. J., Mancino, C. F and Husman, S. H., "Automated irrigation systems using plant and soil sensors", American Society of Agricultural Engineers, ASAE Publication, 1990, pp. 533-537.

4. Benzekri, A., Meghriche, K., and Refoufi, L., PCbased automation of a multi-mode control for an irrigation system Proceedings of International symposium on industrial embedded systems, Lisbon, July 2007, pp. 310-315.

5. Shinghal, K., Noor, A., Srivastava, N., and Singh, R., Wireless sensor networks in agriculture for potato farming International Journal of Engineering, Science and Technology, Vol. 2, No. 8, 2010, pp. 3955-3963.

6. [56Gautam, I,, and Reddy, S. R. N., Innovative GSM-Bluetooth based remote controlled embedded system for irrigation, International Journal of Computer Applications, Vol. 47, No. 8, 2012, pp. 1.

7. Zhang, F., Yang, M., and Ying, H., The application of GSM communication in agricultural automation, Journal of Technology for Agriculture, Vol. 1, No. 1, 2004, pp. 39-41.

8. Shen Jin, Song Jingling, Han Qiuyan, Wang Shengde, and YangYan, School of Electric and Electronic Engineering, A Remote Measurement and Control System for Greenhouse Based on GSM-SMS, IEEE 8th International Conference on Electronic Measurement and Instrument, 2007,pp. 45-82

9. Webin Huang, Guanglong Wang, Research of Wireless Sensor Networks for an Intelligent Measurement System Based on ARM,pp.10741079, 2011.

10. Sezen S M, Yazar A, Irrigation Management on Yield And Quality Of Tomatoes Grown in different Soilless Media in Glasshouse,41-48, 2010 .

11. Daniel K. Fisher and Hirut Kebede, a Low Cost Microcontroller-Based System to Monitor Crop Temperature and Water Status, pp.168-173, 2010. 